\title{
Des frères... presque jumeaux : les Pères blancs belges et canadiens-français entre colonisation et mission dans l'Afrique des années 1950
}

\section{Catherine Foisy}

Volume 67, numéro 3-4, hiver-printemps 2014

URI : https://id.erudit.org/iderudit/1030036ar

DOI : https://doi.org/10.7202/1030036ar

Aller au sommaire du numéro

Éditeur(s)

Institut d'histoire de l’Amérique française

ISSN

0035-2357 (imprimé)

1492-1383 (numérique)

Découvrir la revue

Citer cet article

Foisy, C. (2014). Des frères... presque jumeaux : les Pères blancs belges et canadiens-français entre colonisation et mission dans l'Afrique des années 1950. Revue d'histoire de l'Amérique française, 67(3-4), 295-315.

https://doi.org/10.7202/1030036ar

\section{Résumé de l'article}

Cet article va au-delà des statistiques missionnaires belges et québécoises en comparant les revues publiées par les Pères blancs belges et

canadiens-français, tant du point de vue de leurs relations avec leurs coreligionnaires nationaux que de leur action missionnaire en territoire africain au cours des années 1950. Il explore d'abord les stratégies de propagande, de financement et de recrutement mises en place pour maintenir l'intérêt, l'engagement et le support des catholiques belges et

canadiens-français face aux missions étrangères. Par la suite, il revisite l'action missionnaire de ces deux groupes dans divers pays africains en se focalisant sur les dimensions politiques internes au catholicisme telles que déployées en mission et sur les liens entretenus par les missionnaires avec les empires coloniaux. Enfin, il s'emploie à dégager les principales similitudes et différences entre les deux cas, montrant que les deux groupes étudiés se ressemblent davantage qu'ils se distinguent, malgré une différence majeure, celle d'appartenir à une nation coloniale.
Tous droits réservés (C) Institut d’histoire de l'Amérique française, 2015
Ce document est protégé par la loi sur le droit d'auteur. L'utilisation des services d’Érudit (y compris la reproduction) est assujettie à sa politique d'utilisation que vous pouvez consulter en ligne. 


\title{
Des frères... presque jumeaux : les Pères blancs belges et canadiens-français entre colonisation et mission dans l'Afrique des années I950
}

\author{
CATHERINe FoisY ${ }^{1}$ \\ Département des sciences de la religion \\ Université du Québec à Montréal
}

RÉSUMÉ - Cet article va au-delà des statistiques missionnaires belges et québécoises en comparant les revues publiées par les Pères blancs belges et canadiens-français, tant du point de vue de leurs relations avec leurs coreligionnaires nationaux que de leur action missionnaire en territoire africain au cours des années 1950. II explore d'abord les stratégies de propagande, de financement et de recrutement mises en place pour maintenir l'intérêt, l'engagement et le support des catholiques belges et canadiens-français face aux missions étrangères. Par la suite, il revisite l'action missionnaire de ces deux groupes dans divers pays africains en se focalisant sur les dimensions politiques internes au catholicisme telles que déployées en mission et sur les liens entretenus par les missionnaires avec les empires coloniaux. Enfin, il s'emploie à dégager les principales similitudes et différences entre les deux cas, montrant que les deux groupes étudiés se ressemblent davantage qu'ils se distinguent, malgré une différence majeure, celle d'appartenir à une nation coloniale.

ABSTRACT - This paper goes beyond statistics of missionary work by comparing the newsletters published by the White Fathers from Belgium and French Canada. The paper considers both the missionaries' relations with their people at home and in the missionary field in Africa in the 1950s. It first explores strategies of propaganda, financing, and recruitment aimed at maintaining Belgian and French-Canadian interest, commitment, and support of foreign missions. Secondly, it analyses the missionary actions of the two groups in different African countries, focusing mainly on their politic interactions within the realm of Catholicism, as experienced in the field, and with colonial empires. Thirdly, the paper brings out their main

1. L'auteure tient à remercier les évaluateurs ainsi que l'équipe éditoriale de la RHAF pour leurs précieux et judicieux commentaires qui ont enrichi le présent article. 
similarities and differences, showing that the two groups have more in common than they have differences, despite the major difference that they respectively belong to colonial and non-colonial nations.

\section{l'aube de la Révolution tranquille, le Canada français est devenu un - joueur incontournable de la "planète» missionnaire catholique,} faisant autant la fierté du peuple canadien-français que celle de son Église ${ }^{2}$. En moins de 60 ans, l'effort missionnaire canadien-français s'est en effet hissé, au prorata de sa population, au quatrième rang avec 1 missionnaire pour 1120 catholiques, derrière la Belgique (1:1050), la Hollande $(1: 566)$ et l'Irlande $(1: 457)^{3}$. Cet article veut aller au-delà de ces statistiques en comparant le Canada français et la Belgique missionnaires, une entreprise jamais tentée à ce jour.

L'historiographie belge compte des travaux sur les périodiques missionnaires de l'époque de l'entre-deux-guerres ${ }^{4}$, puis de celle des années 1970 à aujourd'hui ${ }^{5}$, sur la question scolaire en mission ${ }^{6}$, sur le rôle joué par l'épiscopat en contexte colonial' et sur les enjeux de l'inculturation du message chrétien ${ }^{8}$. De plus, comme le rappelle Marvin D. Markowitz ${ }^{9}$ la figure du missionnaire incarne, dès les débuts du mouvement de colonisation de l'Afrique au cours du dernier tiers du XIX ${ }^{\mathrm{e}}$ siècle, à la fois le bras séculier et spirituel de la domination occidentale en Afrique ${ }^{10}$. Toutefois, il insiste sur la spécificité du cas belge, notamment par rapport aux sys-

2. Lionel Groulx, Le Canada français missionnaire. Une autre grande aventure (Montréal, Fides, 1962).

3. Ce sont les statistiques au début des années 1950. Jean Hamelin, Le XXe siècle, Tome 2: De 1940 à nos jours, dans Nive Voisine, Histoire du catholicisme québécois (Montréal, Éditions du Boréal, 1984), 191.

4. Jean Pirotte, Périodiques missionnaires belges d'expression française. Reflets de cinquante années d'évolution d'une mentalité, 1889-1940 (Louvain, Publications universitaires de Louvain, 1973).

5. Olivier Servais, «Dimension politique de la revue Vivant Univers des Pères blancs belges de 1969 à 2002 ", dans Caroline Sappia et Olivier Servais, dir., Mission et engagement politique après 1945. Afrique, Amérique latine, Europe (Paris, Karthala, 2010), 71-85.

6. Marvin D. Markowitz, Cross and sword the political role of christian missions in the belgian congo, 19081960 (Stanford, Calif. Stanford University, Hoover Institution, 1973), 223 p. Pierre Kita Masandi, «L'éducation féminine au Congo belge ", Paedagogica Historica: International Journal of the History of Education, 40, 4 (2004): 479-508.

7. Flavien Nkay Malu, «L'épiscopat du Congo belge et l'indépendance : la déclaration de 1956 ", dans C. Sappia et O. Servais, dir., op. cit., 273-288.

8. Henri Derroitte et Claude Soetens, La mémoire missionnaire. Les chemins sinueux de l'inculturation (Bruxelles, Lumen Vitae, coll. «Théologies pratiques», 1999).

9. Marvin D. Markowitz, "The missions and Political Development in the Congo », Africa: Journal of the International African Institute, 40, 3 (juillet 1970): 235-236.

10. Selon Côme Kinata, l'attitude des premiers missionnaires est complexe et il s'avère difficile de dissocier le succès de leur mission évangélisatrice de l'affermissement de la colonisation française. Côme Kinata, "Les administrateurs et les missionnaires face aux coutumes au Congo français », Cahiers d'études africaines, 3, 175 (2004): 593-608. 
tèmes coloniaux britannique et français où, après une période de stabilisation administrative, les missionnaires ont perdu de leur pouvoir d'intervention politique au profit d'administrations coloniales séculières. Du côté canadien-français, des travaux récents ont montré l'intérêt de la problématique missionnaire en contexte africain, la période des années 1950 présentant, à plus d'un égard, une pertinence certaine ${ }^{11}$, spécialement afin de saisir la nature des changements opérés au cours de la décennie 1960, marquée par le Concile Vatican II et par la déferlante des indépendances politiques.

Cet article propose une analyse thématique comparative de la revue Grands Lacs devenue en 1957 Vivante Afrique, publiée par les Missionnaires d'Afrique (Pères blancs) belges et de la revue publiée par leurs homologues canadiens-français sous le titre Les Missions d'Afrique au cours de la décennie 1950. L'analyse vise à exposer les principaux défis du terrain missionnaire en Afrique au cours de la décennie et les réponses apportées tant par les Canadiens français que par les Belges. Dans le contexte des années 1950 marquées par une professionnalisation de la formation des religieuses missionnaires ${ }^{12}$, une attention particulière sera également portée à la place faite aux religieuses missionnaires dans ces pages ainsi qu'aux questions de développement relatives à la gent féminine africaine.

En tant que nations ${ }^{13}$ de taille similaire, toutes deux au faîte de leur action missionnaire au cours de la décennie 1950, actives sur un continent encore fortement conditionné par la présence coloniale, la Belgique et le Canada français présentent deux cas pertinents pour étudier la manière dont se déploient et évoluent rapidement les rapports entre religion et politique sur le terrain missionnaire africain, et ce, sur deux versants principaux. D'une part, ce sera une occasion de s'attarder aux dynamiques politiques internes au catholicisme, telles que manifestées dans les rapports de force

11. Jean-Philippe Warren, «Les commencements de la coopération internationale Canada-Afrique. Le rôle des missionnaires canadiens ", dans Jean-Bruno Mukanya Kaninda-Muana, Les relations entre le Canada et l'Afrique depuis 1960 (Paris, L'Harmattan, 2012), 23-49. Éric Desautels, «Échanges, adaptations et traductions dans l'histoire missionnaire: les Sœurs blanches du Canada français (1903-2013)", Études d'histoire religieuse, 80, 1-2 (2014): 43-62.

12. Catherine Foisy, Des Québécois aux frontières: dialogues et affrontements culturels aux dimensions du monde. Récits missionnaires d'Asie, d'Afrique et d'Amérique latine (1945-1980), Thèse de doctorat (Humanities), Université Concordia, 2012, 95-115.

13. Je considère le Canada français comme une nation puisqu'il renvoie à la fois à un territoire ainsi qu'à un groupe de population l'habitant et partageant des caractéristiques et des institutions culturelles, économique, historiques et politiques communes, éléments qui participent de la définition classique d'une nation. 
entre missionnaires féminins et masculins, entre instituts missionnaires et autorité vaticane de même qu'entre Église romaine et Églises locales africaines. D'autre part, les relations entre les États coloniaux et l'Église (autorités vaticanes, épiscopales et simples missionnaires) retiennent l'attention dans la mesure où elles permettent notamment de mesurer les impacts des confrontations entre des logiques nationale et supranationale alors en jeu dans les missions catholiques. Autrement dit, l'ambition de cet article est de répondre à une question simple : quel rôle l'appartenance nationale de Pères blancs belges et canadiens-français actifs en Afrique dans les années 1950 a-t-elle joué sur leur lecture des transformations alors en cours (affrontements idéologiques, volonté de souveraineté politique face au colonialisme) ainsi que sur leurs pratiques (éducation, services socio-sanitaires, mouvements de jeunesse, évangélisation), telles que traduites dans leurs revues respectives? Je fais l'hypothèse que cette appartenance fut déterminante, conduisant à des lectures et des pratiques missionnaires marquées principalement par la différence.

Pour mieux cerner ces diverses réalités, cet article explore, dans un premier temps, les stratégies de propagande mises en place pour maintenir l'intérêt, l'engagement et le support des catholiques belges et canadiensfrançais face aux missions étrangères. Dans un second temps, il revisite l'action missionnaire des Pères blancs belges et canadiens-français, telle qu'eux-mêmes l'articulent et la représentent dans leurs revues respectives au cours de la décennie 1950, en prenant soin de contextualiser spécifiquement l'action et le discours de l'Église à l'endroit des nations africaines. Enfin, à partir des principales similitudes et différences entre les deux cas, il montre à quel point le politique et le religieux sont demeurés, à travers les missions en Afrique, intimement liés, et ce, tant sur le plan de la vie interne de l’Église catholique que sur celui des rapports entre État et Église.

\section{LES STRUCTURES D'APPUI AUX MISSIONS CATHOLIQUES CANADIENNES-FRANÇAISES ET BELGES}

Les Canadiens français, autant que les Belges, ont accès à une foisonnante littérature missionnaire par l'entremise des revues que publient les instituts religieux. Alors que les premières publications belges datent de la deuxième moitié du XIX ${ }^{\mathrm{e}}$ siècle, les premiers bulletins relatifs aux missions étrangères publiés au Québec remontent à 1895 avec les Missionnaires d'Afrique et les Franciscains ${ }^{14}$. Dans les années 1950, on dénombre au Québec pas moins 
de 45 titres différents de revues missionnaires, un chiffre équivalant à ce que l'on retrouve alors en Belgique. Ces revues jouent un triple rôle: faire connaître les œuvres des instituts, susciter des vocations missionnaires parmi les jeunes qui en prennent connaissance et favoriser la participation la plus élargie possible du public catholique aux missions étrangères en les sensibilisant aux besoins des instituts et des peuples de mission.

D’ailleurs, les procédés qu'emploient les instituts missionnaires sont pratiquement les mêmes, en Belgique et au Canada français: récits de vocations missionnaires, exaltation de la vie trépidante d'aventures des missionnaires, mise en récit de conversions et, par ce fait, vibrant plaidoyer rappelant la nécessité d'apporter la lumière de la foi chrétienne à des populations jugées être restées païennes jusqu'à ce jour. Un second médium particulièrement en vogue au cours de la décennie 1950, les films, font également partie de l'arsenal de propagande missionnaire dont disposent les instituts canadiens-français et belges. Là aussi, cependant, l'aventure commence plus tôt dans le second cas, alors que les premiers films sont tournés dès le début des années $1920^{15}$. Dans le cas canadienfrançais, il faudra attendre la toute fin de cette décennie et surtout, le début des années 1930 pour que des missionnaires tournent leurs premiers films ${ }^{16}$ et que ces derniers soient diffusés à travers les tournées dans les collèges, les écoles et les expositions missionnaires tenues dans la belle province.

Ces outils de propagande contribuent aux succès des entreprises missionnaires, notamment par le truchement des ressources financières et matérielles que les instituts sont ainsi en mesure de dégager. Par exemple, en 1950, l'œuvre de la Sainte-Enfance au Canada français a récolté 199135 \$ et 204288 \$ l'année suivante, alors qu’en 1955, 1956 et 1957, les Euvres de Saint-Pierre Apôtre et de la Propagation de la Foi disposent de fonds cumulés de plus d'un million de dollars ${ }^{17}$. Bien que je n'aie pas été en mesure

15. Flavien Nkay Malu, «Le cinéma au service de l'évangélisation: les Scheutistes au Congo belge», dans Émilie Gangnat, Annie Lenoble-Bart et Jean-François Zorn, dir., Mission et cinéma: Films missionnaires et missionnaires dans le cinéma (Paris, Karthala, 2013), 111-127.

16. Il semble que les premiers films produits par des missionnaires québécois datent de la fin des années 1920 et des années 1930. Par exemple, une série de films a été présentée lors de l'Exposition missionnaire de 1942 célébrant le tricentenaire de Montréal et tenue à l'Oratoire Saint-Joseph du 17 septembre au 4 octobre 1942 dont ceux des Frères de Sainte-Croix intitulés «Les Missionnaires de SainteCroix au Bengale» et «Dans la jungle». Catherine Foisy, «Les missions québécoises à travers le cinéma : un regard socio-ecclésial en mutation (1942-1990)», dans É. Gangnat, A. Lenoble-Bart et J.-F. Zorn, dir., op. cit., 129-141.

17. Les chiffres détaillés sont, en 1955, de 353851 \$ pour l'œuvre de Saint-Pierre apôtre et de 655000 \$ pour la Propagation de la foi; en 1956, respectivement de 415095 \$ et de 712940 \$ puis en 1957, respec- 
d'établir des chiffres similaires pour la Belgique, une différence notable dans le financement des missions catholiques belges tient aux subsides importants versés par l'administration coloniale, une forme de sous-traitance étatique. Le concordat signé en 1906 entre l'État indépendant du Congo et le SaintSiège stipule que des subsides doivent être versés par l'administration coloniale aux écoles dirigées par des missionnaires catholiques. De plus, l'État s'engagea alors à accorder à toute nouvelle mission catholique un droit perpétuel sur un terrain de 100 à 200 hectares $^{18}$. En contexte français, les allocations du gouvernement aux écoles tenues par des missionnaires sont insuffisantes et les missions sont soumises «aux tarifs douaniers et impôts sur les terres et produits domaniaux au même titre que le commun des colons ${ }^{19}$ ", ce qui ne sied pas aux missionnaires. Par ailleurs, dans tous les cas de figure, notons que la main-d'œuvre religieuse, largement féminine, est cependant peu rétribuée ${ }^{20}$.

Enfin, dans le domaine que je qualifierais «d'intelligence des missions $^{21}$ ", les Belges avaient pris une avance sur les Canadiens français en lançant les Semaines de missiologie à l'Université catholique de Louvain, sous le leadership du Jésuite Pierre Charles, dès $1923^{22}$. Cette initiative s'inscrit d'ailleurs dans la foulée des encycliques missionnaires Maximum Illud (1919) et Rerum Ecclesice (1926) auxquelles est également associé le renouveau des hautes études missionnaires dont témoigne la fondation de chaires tant à la Grégorienne (1932) qu'à l'Urbaniana (1933). Il faudra attendre 1934 pour que s'organise la première Semaine d'études missionnaires au Québec ${ }^{23}$, puis 1948 pour qu'une chaire en sciences de la mission

tivement de 479122 \$ et de 751000 \$. Compilations établies par Jean-Philippe Warren pour l'Atlas missionnaire sous la direction de Gilles Routhier et de Frédéric Laugrand (ouvrage en préparation).

18. M. D. Markowitz, loc. cit., 236-237.

19. C. Kinata, loc. cit., 595.

20. M. D. Markowitz, op. cit. Des religieuses missionnaires canadiennes-françaises actives au Congo belge dans les années 1950 étaient employées par des entreprises coloniales et payées en partie par l’État colonial belge, soit pour des fonctions éducatives ou socio-sanitaires, notamment au sein de centres extra-coutumiers.

21. Ce n'est qu'en 1910 que l'historien de l'Église, l'Allemand Josef Schmidlin, commence à établir les bases de la discipline de la missiologie et à l'enseigner à la Faculté catholique de théologie de Münster.

22. Jean Pirotte, "D’une missiologie "confessante" à une missiologie "distanciée” ? Les études missiologiques en Belgique au XX ${ }^{\mathrm{e}}$ siècle», Histoire, monde et cultures religieuses, 1 (2007): 51-62.

23. Fondée par le père Léo Deschâtelets, omi, la première semaine d'études missionnaires a lieu en octobre 1934 à Ottawa sur le thème «Introduction aux problèmes des missions » et regroupe diverses communautés religieuses québécoises actives à l’extérieur du pays. En 1936, se réunissent à Québec des membres de congrégations pour discuter de ce thème "La conversion des Infidèles", puis en 1949 à Québec encore, pour s'entretenir du thème «La valeur religieuse des religions païennes et l'adaptation missionnaire » puis à Montréal en 1950 autour de ce thème «Le laïcat et les missions ». 
soit créée à l'Université d'Ottawa ${ }^{24}$. Les Oblats de Marie-Immaculée, qui prennent la tête de ce mouvement au Canada, jouent également un rôle de leader sur le plan de la connaissance en mission, notamment avec la fondation de l'Aide intellectuelle missionnaire au cours des années 1930 et dont les fruits sont nombreux pour l'Afrique au cours de la décennie $1950^{25}$.

\section{UN MODÈLE «CIVILISATEUR » SUR FOND DE COLONIALISME ET DE SUPRANATIONALISME}

De mon point de vue, quatre éléments principaux permettent de contextualiser la dynamique propre de l'effort missionnaire catholique en Afrique au cours de la décennie 1950. Premièrement, la géopolitique mondiale est agitée en cette période de Guerre froide et les missions catholiques sont ressorties particulièrement ébranlées de l'aventure chinoise $^{26}$. La prise du pouvoir par les communistes en 1949 a très fortement marqué les consciences et renforcé la position du Vatican à l'égard de cette idéologie politique que Pie XII n'aura de cesse de dénoncer dans ses deux encycliques missionnaires (Evangelii Praecones, $1951^{27}$ et Fidei Donum, $\left.1957^{28}\right)$.

Deuxièmement, le discours de reconnaissance des droits humains, garants de l'égale dignité de chacun et de chacune, peu importe sa race, son opinion politique ou autre, trouve un écho de plus en plus grand dans l'enseignement officiel de l’Église au cours de la décennie ${ }^{29}$. Il représente également une justification séculière des interpellations lancées, dans les encycliques

24. D’ailleurs, le chef-lieu du développement des sciences de la mission au Canada se concentrera à l'Université Saint-Paul d'Ottawa, lequel connaîtra son véritable essor au cours des années postconciliaires. Notons que le Centre d'études missionnaires fondé par le père Jean Bouchard, sj, en 1958 sera également un lieu de formation important pour les instituts missionnaires québécois, lequel prendra le nom de Centre d'étude et de coopération internationale (CECI) en 1967 au moment de sa sécularisation.

25. Les statistiques de l'Aide intellectuelle missionnaire, publiées dans L'Apostolat de janvier 1958, indiquent que depuis 25 ans, les dons des Canadiens ont pu fournir à l'Afrique 16680 volumes, 17453 volumes à l'Asie, 9450 volumes au Canada ("missions esquimaudes et indiennes») et 2245 volumes en Amérique du Sud, où la présence des OMI est beaucoup plus récente (après 1945).

26. Pour le cas québécois, voir C. Foisy, Des Québécois..., op. cit., 116-150.

27. L'objet principal de cette encyclique est l'appréciation des cultures et des religions, mais elle vise aussi à intéresser les fidèles à certains territoires de mission qui n’apparaissent pas comme évidents au premier abord, l'Amérique latine, par exemple. Selon Pie XII, privilégier l'Amérique latine dans la poursuite des entreprises missionnaires catholiques, même si la région est déjà christianisée, permettrait principalement de faire contrepoids à la poussée des Églises protestantes, à la diffusion du matérialisme athée et de la franc-maçonnerie.

28. Cette deuxième encyclique missionnaire de Pie XII favorise une participation des Églises diocésaines d'Occident en faveur des «jeunes Églises» du Sud (en particulier celles d'Afrique).

29. Claude Prudhomme, Missions chrétiennes et colonisation, XVI ${ }^{e}$-XX $X^{e}$ siècles (Paris, Cerf, 2005). 
missionnaires, à tous les catholiques (incluant les laïques) afin qu'ils s'engagent sur le terrain missionnaire notamment en Afrique.

Troisièmement, au cours des années 1950, et cela est une conséquence des deux éléments précédents, une part toujours plus large est faite à la formation d'une élite catholique africaine, notamment par le développement d'institutions d'enseignement supérieur, non sans heurts et difficultés, spécialement en contexte belge où les confrontations avec l'État colonial émergent dès $1945^{30}$ pour se cristalliser, comme nous le verrons sous peu, autour de la question scolaire dans sa globalité dès 1954 .

Quatrièmement, les années 1950 sont le moment où s'officialise une politique vaticane entamée dès le début du $\mathrm{XX}^{\mathrm{e}}$ siècle, celle de l'autochtonisation des Églises dites de mission. En effet, Rome nomme, au cours de la décennie 1950, près d'une vingtaine d'évêques africains à la tête de diocèses ou de vicariats apostoliques du continent noir, information relayée par les deux revues missionnaires consultées ${ }^{31}$.

Quel est le portrait du clergé dans les missions africaines au début des années 1950? D’abord, 19,7\% du clergé missionnaire en Afrique est belge (1583) contre 3,3\% pour les Canadiens (282 $)^{32}$. Alors que les Belges se concentrent en Afrique centrale $(1523)^{33}$, là où se trouvent leurs colonies (Congo et Ruanda-Urundi), les Canadiens sont principalement stationnés en Afrique méridionale (114) et orientale (123), soit dans des pays sous contrôle britannique (notamment, le Basutoland, l'Afrique du Sud, l'Ouganda, le Tanganyika britannique ${ }^{34}$, la Rhodésie du Nord et le NyassaNord). N'oublions pas qu'un Canadien français est alors un citoyen de l'Empire et réputé plus à même de maîtriser l'anglais.

La décennie 1950 est celle d'une croissance importante de la présence missionnaire canadienne-française en Afrique. Selon Lionel Groulx, si on dénombre 925 missionnaires en 1953, ils sont 1500 appartenant à 48

30. M. D. Markowitz, loc. cit., 239-240.

31. Raymond Lacroix, "Échos d'un grand jour. Sacre de Son Excellence Monseigneur Laureano Rugambwa (Lettre à des petites croisées)", Les Missions d'Afrique (août 1952): 229-236. Vincent de Decker, "La mission d'Afrique», Vivante Afrique, 196 (avril-mai 1958). 32 p.

32. Ce sont les chiffres de l'année 1949. La rédaction, "Clergé dans les missions africaines », Grands Lacs, 152 (mai 1952): 36.

33. Comme l'indique William Blondeel: «[...] en date du 30 juin 1959, la proportion des prêtres non belges, par rapport à la totalité des missionnaires-prêtres au Congo (2308), n'est que de 1 sur 5, tandis que celle du Ruanda-Urundi s'élève à 1 sur 3 (113 sur 338) (chiffres calculés sur la base de l'Annuaire Catholique 1960-1961).» Blondeel explique cette différence entre les deux secteurs par leurs statuts administratifs distincts du point de vue colonial; dans le premier cas, la Belgique joue un rôle de métropole alors que dans le second, elle agit comme mandataire. William Blondeel, «Les missionnaires belges en Afrique centrale, fin $19^{\mathrm{e}}-20^{\mathrm{e}}$ siècle. L'Évangélisation: pas un monde à part», Afrika Focus, 2, 1 (1986): 96.

34. De 1919 à 1964, car avant cette date, il fut sous contrôle allemand. 
sociétés religieuses et répartis entre 22 pays $^{35}$ en 1959. Il faut savoir que même si les papes Benoît XV (1914-1922) et Pie XI (1922-1939) ont cherché à imposer le caractère supranational des missions en contexte africain,

[...] ils sont impuissants à empêcher la relève des missionnaires germaniques dans les anciennes colonies allemandes (Cameroun, Togo) par des sociétés missionnaires françaises ou britanniques. Il faut attendre la seconde guerre mondiale pour que se généralise la diversification du recrutement ${ }^{36}$.

C’est donc dans ce contexte de généralisation de la diversification du recrutement missionnaire que se situe cette présence croissante des Canadiens français en Afrique. L'Annuaire catholique de 1960-1961 mentionne, pour sa part, un grand nombre d'entités missionnaires belges en Afrique: 37 de prêtres, 9 de frères et pas moins de 89 instituts religieux féminins ${ }^{37}$. Les Canadiennes françaises ne sont pas en marge du mouvement, étant pour leur part réparties entre 30 communautés de fondation canadienne-française.

Un balayage initial des revues publiées par les Pères blancs belges et canadiens-français nous convainc rapidement que les domaines d'engagement missionnaire les plus significatifs, outre celui de l'évangélisation au sens strict du terme, demeurent, tout au long de la décennie 1950, la santé, l'éducation et les services sociaux, particulièrement à destination des femmes et des enfants. Cette orientation relève d'une structuration interne des missions catholiques où les religieuses missionnaires ont joué un rôle de premier plan ${ }^{38}$, les autorités masculines de l'Église reconnaissant d'ailleurs la nécessité d'une professionnalisation accrue pour ces femmes $^{39}$. Ce déploiement de ressources missionnaires sur le continent africain au cours de cette décennie est aussi l'une des résultantes de la réorganisation des missions catholiques à la suite de l'avènement du communisme en Chine, un mouvement d'ailleurs vivement souhaité et fortement appuyé par Rome.

Par ailleurs, le rythme de l'histoire missionnaire s'accélère au cours de cette décennie, plusieurs facteurs étant en cause, dont le plus déterminant sera la conquête de l'indépendance politique par les nations africaines. Face à cet enjeu qui confronte les missionnaires, tant belges que canadiens-

35. L. Groulx, op. cit., 260.

36. Claude Prudhomme, "Centralité romaine et frontières missionnaires", Mélanges de l'École française de Rome, Italie et Méditerranée, 109, 2 (1997): 487-504.

37. Cité dans W. Blondeel, loc. cit., 93-114.

38. Katharina Stornig, Sisters Crossing Borders. German Missionary Nuns in Colonial Togo and New Guinea, 1897-1960 (Göttingen, Vandenhoeck \& Ruprecht, 2013), 413 p.

39. C'est en 1954 que le pape Pie XII bénit l’Institut Regina Mundi fondé à Rome pour prendre en charge la formation approfondie d'un personnel religieux féminin sur les plans pastoral et théologique. 
français, la décennie voit se transformer le positionnement des uns et des autres, spécialement dans sa deuxième moitié ${ }^{40}$. Ce propos sera illustré par une analyse des principaux thèmes dégagés dans chacune des revues.

D'abord, la revue des Pères blancs belges fait une place importante aux instances administratives coloniales, tout autant qu'elle illustre et défend le projet colonisateur national en contexte congolais:

L'action colonisatrice me parait comporter deux aspects qu'il faut pouvoir dissocier pour en comprendre la portée, mais qui, dans l'action, devraient être constamment réunis : l'aspect économique et l'aspect humain, la colonisation proprement dite et l'action civilisatrice. L'occupation du territoire, son exploitation rationnelle sont une chose, légitime d'ailleurs dans les limites de la raison et de l'humanité; l'action civilisatrice, le progrès, le mieux-être des populations autochtones en sont une autre ${ }^{41}$.

Des Africains, tels que le roi du Ruanda, et certains prêtres congolais prennent la plume dans les pages de Grands Lacs, allant jusqu'à demander aux Européens de se mélanger davantage aux «évolués» congolais et, par ce biais, de mieux participer à l'œuvre "civilisatrice» entreprise en Afrique ${ }^{42}$. D’ailleurs, la revue revient de manière cyclique, au cours des années 1950, sur la préparation et la formation des futurs coloniaux et des futures coloniales, prodiguant divers conseils sur la manière de se comporter au Congo belge, notamment. Des coloniaux eux-mêmes décrivent leurs conditions de vie, montrant bien là l'intégration des missionnaires à la vie coloniale belge, laquelle est, selon Markowitz, littéralement dominée, de la fin du XIX $^{e}$ siècle jusqu'aux années 1950, par ces derniers qui occupent jusqu'aux échelons les plus élevés du ministère belge des Colonies ${ }^{43}$.

La poursuite de l'œuvre «civilisatrice» est d'ailleurs la pierre d'angle du discours missionnaire belge des années 1950 que résume admirablement cet autre extrait de Grands Lacs:

À qui considère l'histoire et en particulier les événements contemporains, le doute n'est pas possible. L'Europe trouve dans la crise profonde qu'elle traverse actuellement une occasion de purifier et de spiritualiser son christianisme. L'avance qu'elle a prise dans le progrès des techniques, lui assure une

40. Éric Desautels montre bien que le discours des missionnaires québécois, particulièrement celui des femmes, se transforme positivement face à cette question politique majeure. Éric Desautels, "La représentation sociale de l'Afrique dans le discours missionnaire canadien-français (1900-1968)", Mens: revue d'histoire intellectuelle et culturelle, 13, 1 (automne 2012): 81-107.

41. J. Spaey, "Assistance sociale et civilisation», Grands Lacs, 130-131 (avril 1950): 68.

42. Édouard Mbala, «Futurs coloniaux, futures coloniales. Blancs et Noirs», Grand Lacs, 166 (octobre 1953): 9-10. Cet article est emprunté à La Voix du Congolais de juillet 1953.

43. M. D. Markowitz, loc. cit., 234-247. 
influence prépondérante sur tous les peuples de l'Asie et de l'Afrique. Le temps marqué par Dieu pour la délivrance de ces peuples est arrivé ${ }^{44}$.

Évidemment, on ne parle pas d'une délivrance politique par les Africains eux-mêmes, mais bien par le biais d'une action "civilisatrice» dont la mission catholique demeure l'instrument le plus sûr ${ }^{45}$. Signes d'un positionnement en cours de transformation par rapport au projet national belge au Congo? Deux extraits remarquables datés de 1952, sont empreints de confiance à l'égard des élites locales et même d'un certain détachement. L'auteur recommande aux missionnaires d' "Accepter que l'évolution puisse se faire sans épouser exactement le moule de nos conceptions européennes et dans le respect des particularismes locaux ${ }^{46}{ }^{\prime}$. L'article se conclut sur ces mots, inspirés par la vision missionnaire du fondateur des Pères blancs, le cardinal Lavigerie:

Le salut de l'Afrique et son élévation ne se feront pas sans les Africains, mais avec eux et par eux. [...] À l'égard de cette élite, deux consignes précises s'imposent: faire confiance et laisser agir ${ }^{47}$.

Pour leur part, les Pères blancs canadiens-français ne sont pas en reste de ce mouvement "civilisateur» comme nous le verrons sous peu, mais ils demeurent plus discrets au sujet des relations qu'ils entretiennent avec les autorités coloniales, du moins dans la revue consultée. Choix stratégique de la province d'un institut missionnaire international dont les membres se retrouvent dans des contextes coloniaux fort différents, où le sentiment national, voire nationaliste, ne joue que peu ou prou? Sentiment empathique des membres d'un peuple qui vit lui-même, dans son propre pays, une situation de colonisé ? En tous les cas, outre les rares mentions de dignitaires coloniaux ou les revendications en faveur de leurs œuvres d'éducation adressées aux administrations coloniales, les Pères blancs canadiens-français demeurent peu loquaces par rapport à leurs relations avec les divers États coloniaux où ils sont engagés.

44. André Lacoste, «Le catholicisme face au monde du XX siècle», Grands Lacs, 150 (mars 1952): 44.

45. D’ailleurs, comme l'indique le père Guy Mosmans: «Pour justifier cette œuvre d'Éducation totale, nous ne faisons donc pas appel aux principes de la civilisation occidentale en tant qu'occidentale, mais aux principes de la civilisation occidentale en tant qu'humaine, mieux: aux principes de civilisation humaine que, grâce à l'Évangile, possède, mieux que toute autre, la civilisation occidentale, c'est-à-dire au droit naturel, seul droit que les divers peuples n'aient pas droit de choisir à leur gré mais auquel tous doivent se conformer. C’est de cette double supériorité, économique et spirituelle, que découlent les principes directeurs de l'éducation civilisatrice. » Guy Mosmans, «Conception chrétienne de la colonisation", Grands Lacs, 150 (mars 1952): 36-37.

46. Guy Mosmans, «Le rôle social de l'enseignement aux indigènes», Grands Lacs, 152 (mai 1952): 20.

47. Ibid., 27. 
En contrepartie, tant les Pères blancs canadiens-français que belges font une place de choix aux messages catholiques officiels provenant du pape, des cardinaux ou des évêques. Outre les récits de vocations et d'expériences missionnaires, la mission y est dépeinte comme une expérience riche de défis et de risques vécus au milieu de populations "païennes». Dans le cas du Canada français, on exalte la mission comme la fine fleur du nationalisme canadien-français. Du côté belge, on nous présente les Noirs comme des mineurs et des gens parfois peu avancés sur le plan technique et matériel, mais également et surtout moral ${ }^{48}$ - un trait sur lequel on insistera d'ailleurs fortement dans la deuxième moitié des années 1950 - justifiant la poursuite de l'œuvre "civilisatrice» de l'Église catholique. Autant dans le cas canadien-français que belge, l'intérêt manifesté pour les pratiques rituelles ou les coutumes de ces peuples vise à illustrer la nécessité de poursuivre le travail de conversion et l'œuvre de «civilisation» qui l'accompagne.

Présents dans les domaines social et sanitaire, les Belges comme les Canadiens français font la part belle et relativement large à leurs consœurs. Cependant, les rapports genrés de domination sont présents sur le terrain missionnaire, peu importe que les religieuses soient belges ou canadiennes-françaises. En ce sens, ils traduisent des rapports de force structurants au sein de l'institution ecclésiale qui renvoient à des dimensions politiques internes du catholicisme. Dans un numéro spécial entièrement dédié aux Dominicaines missionnaires de Namur et à leur travail en faveur du «relèvement» de la femme congolaise, tous les textes sont signés par des hommes ${ }^{49}$, même si le travail décrit est bien celui de femmes, illustrant le rôle de subalternes des religieuses missionnaires ${ }^{50}$. Cette manière de

48. Le paternalisme imprègne d'ailleurs très fortement la revue : «Les éléments spirituels de la civilisation n'ont pas encore imprégné l'âme du Noir. Ses progrès économiques sont en avance - et de très loin - sur son évolution morale», Père Van Roy, "Missions, Sociétés coloniales, Colons », Grands Lacs, 130-131 (avril 1950): 77.

49. Par exemple, c'est le ministre des Colonies, Pierre Wigny qui présente la brochure que consacrent les Dominicaines missionnaires de Namur au relèvement de la femme congolaise dans le vicariat de Niangara. Dans un autre article du même numéro de Grands Lacs, le premier président du Conseil d'État, Victor Devaux, prend bien soin de reprendre des expériences des premiers missionnaires et de l'explorateur Stanley pour montrer le chemin parcouru et qu'il était en quelque sorte dans l'ordre des choses qu'il $\mathrm{y}$ ait une différence entre les hommes et les femmes, mêmes missionnaires. Victor Devaux, «La femme congolaise et la civilisation chrétienne», Grands Lacs, 129 (mars 1950): 8.

50. Ce type d'attitudes vis-à-vis des religieuses est profondément ancré dans les mœurs missionnaires comme en témoigne cette anecdote de la toute fin du XIX ${ }^{\mathrm{e}}$ siècle au sujet des sœurs blanches, installées en 1899 à Kisubi en Ouganda: "Tout devait venir d'Europe, par caravane. [...] Les Sœurs racontent comment, après l'ouverture d'une toute petite boutique par un Indien, elles s'y rendirent pour acheter quelques objets. Son Excellence les avait réprimandées pour avoir ainsi été dans un magasin, chez un inconnu, sans avoir sollicité sa permission épiscopale.» Cela en dit beaucoup sur leur niveau d'autonomie 
procéder est en conformité avec une idéologie prégnante sur le terrain missionnaire catholique depuis le dernier tiers du XIX ${ }^{\mathrm{e}}$ siècle et selon laquelle les femmes seraient plus à même de s'approcher de la culture des peuples missionnés par l'établissement de liens de proximité avec les familles ${ }^{51}$. Par exemple, en se remémorant l'inauguration de l'École professionnelle et Ménagère que dirigent les Dominicaines de Namur à Watsa, l'auteur s'interroge: «Comment, en présence de pareils souvenirs, résister à la joie de rendre hommage au travail de celles qui collaborent aussi efficacement à notre splendide ouvre de colonisation ${ }^{52}$ ?» La revue belge rend également compte du travail législatif allant dans le sens de la reconnaissance du mariage chrétien monogamique en cours durant les années 1950. Toutefois, comme le démontre l'historien congolais Pierre Kita Masadi, les femmes sont venues plus tard à l'éducation primaire et secondaire formelle et dans de moins bonnes conditions que leurs homologues masculins ${ }^{53}$.

Pour leur part, les Canadiennes françaises ne sont guère mieux positionnées au sein de l'appareil missionnaire catholique alors qu'elles ont aussi maille à partir avec les autorités épiscopales en de nombreuses occasions. Par exemple, en réponse aux besoins grandissants de la mission de Rulenge (Tanganyika), après de vives discussions avec l'évêque, sœur Annette Roberge, Sœur missionnaire de Notre-Dame des Anges, va finalement chercher quatre jeunes femmes, qui s'installent avec les sœurs à la mission ${ }^{54}$ et qui, au fil du temps, constituent une communauté religieuse autochtone. Dans un article de 1955, le Père blanc québécois Laurent St-Pierre, se plaignant de l'aide reçue de son personnel féminin local, rappelle:

[qu']Il y a une solution, simple comme bonjour, qui nous tirerait d'embarras en un tour de main, ce serait de recevoir ce soir même un contingent de Religieuses indigènes ${ }^{55}$.

Autrement dit, un personnel adéquatement formé selon les termes de l’Église et conformément docile serait préférable à celui que l’on trouve

et le pouvoir que s'arrogent alors les hommes d’Église face à elles. Sœur M. Jean Félix, «Les Sœurs blanches», Grands Lacs, 128 (février 1950): 56.

51. Sarah A. Curtis, "À la découverte de la femme missionnaire", Histoire, monde et cultures religieuses, 4, 16 (2010): 5-18. Elizabeth A. Foster, “"En mission, il faut se faire à tout”. Les Sœurs de l'ImmaculéeConception de Castres au Sénégal, 1880-1900», Histoire, monde et cultures religieuses, 4, 16 (2010): 73-108.

52. Pierre Wigny, «Message de M. Pierre Wigny, Ministre des colonies», Grands Lacs, 129 (mars 1950): 2-3.

53. P. Kita Masandi, loc. cit., 479-508.

54. La rédaction, «Nouvelle entreprise missionnaire», Les Missions d'Afrique (mars 1953): 278-279.

55. Laurent St-Pierre, «Mon personnel féminin», Les Missions d'Afrique (septembre 1955): 281. 
parmi les femmes africaines laïques. Par ailleurs, lors de la première rencontre de dirigeants laïques en Afrique, en 1953, les revues belge et québécoise des Pères blancs ne font aucune mention des propos tenus par les femmes ayant adressé la parole à l'assemblée, bien que les questions relatives à la femme africaine aient été à l'ordre du jour, selon ces deux mêmes sources. Il semble que chacune des publications a davantage retenu comme étant importants les enjeux relatifs aux combats idéologiques à mener contre le marxisme, l'Islam, le protestantisme et le paganisme.

Dans ce contexte, les presses missionnaires, auxquelles participent beaucoup des pères canadiens-français ${ }^{56}$, sont mises à profit pour l'évangélisation des populations africaines, spécialement des élites. Les missionnaires, tant canadiens-français ${ }^{57}$ que belges, $\mathrm{y}$ voient là un devoir, celui

$[\ldots]$ de fournir à nos intellectuels, qui se multiplient rapidement, des livres de religion, plus avancés, des journaux catholiques, des romans chrétiens, des revues techniques, des manuels scolaires, historiques, scientifiques, en un mot de leur fournir une nourriture intellectuelle et morale conforme au sens et à l'esprit que nous voulons leur voir prendre $\mathrm{e}^{58}$.

Dans le même sens, le syndicalisme chrétien est pensé, notamment par les Pères blancs belges en contexte congolais, comme un repoussoir aux tentations du communisme, mais il a besoin d'être solidement renforcé par une culture catholique profondément ancrée dans les mœurs et le quotidien de la jeunesse africaine, en particulier. Ce sera le rôle joué par une panoplie d'actions de nature culturelle, économique, éducative et sociale, toutes inspirées de la doctrine sociale de l'Église catholique. Des initiatives telles que les mouvements d'Action catholique spécialisée, la Légion de Marie, les Ligues du Sacré-Cour, le scoutisme et le guidisme ${ }^{59}$ ainsi que les coopératives de producteurs où les Canadiens français font notamment appel à l'expertise développée à l'Institut Coady d'Antigonish

56. «Où ne se porte point le zèle des missionnaires? L'apostolat de l'imprimé entre largement dans leurs entreprises. Ils impriment leurs livres de classe et de religion, des tracts, des brochures. Ils se font traducteurs en langues africaines d'ouvrages de spiritualité, destinés à leurs convertis, à leurs 2000 frères et sœurs indigènes. Ils publient des journaux: The African, au Nyassaland; Katoliki, au Tanganyika; Munno en Ouganda. Plusieurs Pères blancs canadiens sont engagés en cette œuvre de presse.» (L. Groulx, op. cit., 267)

57. «En 1954, M ${ }^{\text {gr }}$ Alfred Lanctot des Pères Blancs, évêque de Bukoba, pouvait dire à des dirigeants laïques: "Il n'est pas possible que nos élites ne songent pas à avancer, dans toute la mesure du possible, le jour où elles pourront prendre en mains les rênes de l'administration de leur pays. Cette aspiration est naturelle. L’Église ne la désapprouve pas, au contraire.” " (Ibid., 259.)

58. Robert Chaput, "Adaptation missionnaire», Grands Lacs, 130 (mars 1951): 16.

59. Les Pères blancs canadiens-français y sont engagés. Lionel Grandmaison, «Le scoutisme en Ouganda ", Les Missions d'Afrique, (septembre 1953): 231-234. 
en Nouvelle-Écosse ${ }^{60}$, visent à contrer les avancées du matérialisme athée, la résurgence du paganisme, mais aussi la poussée des missionnaires protestants ou les développements de l'Islam ${ }^{61}$. Effet de leur engagement en territoire missionnaire sous contrôle britannique principalement, la confrontation aux missions protestantes fut le lot quotidien de nombreux Pères blancs canadiens-français comme l'indique cet extrait de Les Missions d'Afrique:

Depuis mon dernier appel au secours, la "British and Foreign Bible Society" a mis en vente, au prix très bas de $0,85 \mathrm{cts}$, la Bible complète nouvellement traduite en bemba... [...] Quelle misère que cette vision de la chrétienté! Tout de même, la confusion entre missionnaire et missionnaire n'est pas complète; le catholique fait figure à part. Il a acquis la réputation d'avoir un zèle plus ardent, mieux organisé. Chez nous, il y a une autorité qui commande. Et puis, nos contacts avec les âmes sont autrement plus fréquents, plus intimes, que ceux des Pasteurs. Tout ceci vaut sur le plan humain. Ajoutez que le SaintEsprit doit bien avoir une préférence pour nous et vous comprendrez finalement que nous soyons en train de prendre le dessus, que l'Église catholique s'affirme comme le groupe le plus solide en ce pays ${ }^{62 .}$

Effet inattendu du travail mené dans le cadre de ces œuvres, la seconde moitié de la décennie voit les Pères blancs canadiens-français commencer à remettre en question la valeur positive de la civilisation, identifiant des effets négatifs sur les populations rurales, notamment ${ }^{63}$.

Selon une analyse du rôle politique des missions chrétiennes au Congo belge de 1908 à $1960^{64}$, c'est l'enjeu de l'éducation et des "évolués ${ }^{65}$ " qui divisera le plus les missionnaires belges au cours des années 1950. Il est vrai que la décision de $1954^{66}$ de la coalition gouvernementale libérale-socialiste

60. Voir L. Groulx, op. cit., 276.

61. "Ce prosélytisme déferle sur l'Afrique au moment où les Noirs veulent se libérer de l'animisme et cherchent une autre religion. Se faire chrétien exige la conversion des mours et l'effort de longues années de préparation. [...] Dominé par le sentiment de son impuissance contre son destin, le Noir se retrouve dans le fatalisme musulman.» Roger Colleret, «L'Afrique de demain sera-t-elle chrétienne ou musulmane?", Les Missions d'Afrique (août 1957): 244.

62. Yvan Goulet, «Pas facile de rivaliser! Nouveau Testament», Les Missions d'Afrique (juillet 1957): 220.

63. La rédaction, "Civilisation à rebours ou les effets désorganisateurs du progrès arrivant au fond de la brousse africaine", Les Missions d'Afrique (septembre 1956): 266-285.

64. M. D. Markowitz, op. cit.

65. Les Pères blancs québécois s'en préoccupent également dans ces termes: Maurice Pilon, «Aux prises avec l'évolution", Les Missions d'Afrique (janvier 1958): 15-17.

66. Fait à noter, la même année est fondée l’Université Lovanium à Léopoldville dont les racines se trouvent en Belgique, plus spécialement dans l'Association universitaire catholique pour l'aide aux missions (AUCAM) : «L'AUCAM avait été à l'origine de la création en 1926 de la FOMULAC (Fondation médicale de l'Université de Louvain au Congo) et en 1932 de la CADULAC (Centres agronomiques de 
et anticléricale belge, de fonder des écoles laïques au Congo en remplacement des écoles catholiques dirigées par des missionnaires, allait chambouler le positionnement de nombreux missionnaires belges par rapport au colonialisme ${ }^{67}$. Tout au long de la décennie, de nombreux articles ${ }^{68}$ abordent la question scolaire dans les revues missionnaires canadiennes-françaises, et ce, à tous les niveaux de formation, incluant l'université. Dès 1953, les Pères blancs canadiens-français reviennent sur la position névralgique de la question scolaire pour les missions catholiques:

D'où vient cette importance des écoles en territoires missionnaires? Elle vient des mêmes sources que par ici. L'enfance étant l'âge malléable, apte à recevoir les principes qui auront force de loi plus tard, il importe que cette enfance soit bien orientée; sa volonté sera solide et efficace en autant qu'elle sera basée sur des connaissances plus approfondies et plus étendues. [...] Mais l'Afrique d'aujourd'hui demande plus que des simples écoles de lecture et d'écriture. Elle n'est plus le continent mystérieux, inconnu, d'autrefois. Toutes les croyances, tous les systèmes se font la lutte pour capter le plus possible d'adeptes. On y déverse des sommes énormes, on y envoie des techniciens de première valeur; tout cela pour attirer les populations ${ }^{69}$.

\section{Influencés également par la position favorable du Vatican à la recon-} naissance du droit à l'auto-détermination des peuples africains, surtout à partir de l'encyclique missionnaire Fidei Donum de 1957, les missionnaires belges seront plus que jamais divisés sur la conduite à suivre en regard de

l'Université de Louvain au Congo). Premiers jalons d'un travail louvaniste au Congo, ces institutions furent les embryons de l'Université Lovanium, créée en 1954 à Léopoldville», Jean Pirotte, «D’une missiologie "confessante" à une missiologie "distanciée" ? Les études missiologiques en Belgique au XX" siècle", Histoire, monde et cultures religieuses, 1, 1 (2007): 53.

67. D'ailleurs, le positionnement officiel de l'épiscopat du Congo belge et du Ruanda-Urundi sur cette question changera de manière draconienne avec la publication d'une déclaration favorable à l'indépendance politique de ces peuples, le 29 juin 1956.

68. «De nos jours, la grande préoccupation dans nos missions, c'est la question scolaire: [...] Nos missions d'Afrique, du moins celles du centre et de l'ouest, sont toutes en territoires de colonies et l'influence des pays colonisateurs se fait de plus en plus sentir. Avec cette influence, croît une certaine appréhension, parfois une angoisse : où allons-nous? À quoi nos noirs de demain ressembleront-ils? [...] Et voici le point absurde: tous les gens sérieux des diverses administrations coloniales s'accordent pour reconnaître l'efficacité de notre formation tant au point de vue moral qu'intellectuel. Les élèves instruits dans nos stations coûtent aux Gouvernements dix fois moins cher que ceux instruits dans leurs écoles. Malgré cela, on nous refusera souvent d'ouvrir de nouvelles écoles pendant que des écoles laïques ouvriront leurs portes à grand renfort d'argent. [...] La lutte scolaire est particulièrement aiguë en Afrique Belge, où le ministre des colonies, M. Buisseret, fonde à grands frais un réseau d'écoles laïques au détriment des écoles de Mission. Si les subsides du Gouvernement accordés jusqu’ici aux Missions diminuent ou viennent à manquer, les écoles devront disparaître », Gaston Mathieu, "Un point obscur, absurde», Les Missions d'Afrique (janvier 1956): 14-15.

69. La rédaction, "L'avenir de l'Afrique. Les Pères Blancs d'Afrique, une société missionnaire. À l'œuvre: la question scolaire», Les Missions d'Afrique (novembre 1953): 271. 
l'œuvre colonisatrice de leur nation. Au sein de l'Église, la fin de la décennie voit se matérialiser les aspirations des peuples africains, ce dont rend compte Vivante Afrique:

La remarquable promotion de la hiérarchie africaine se pose comme un fait capital dans l'Église contemporaine. En sept ans, vingt prêtres africains furent promus à la dignité épiscopale. Il ne faudra pas sept ans, sans doute, pour doubler ce nombre. Il y a là, non seulement consécration d'une maturité acquise de la part des chrétientés locales et de leurs chefs spirituels, mais, quant à l'action missionnaire, engagement effectif dans une voie longtemps préparée et définitivement ouverte. Une ère nouvelle marque la Mission d'Afrique ${ }^{70}$.

Pour sa part, la revue des Pères blancs canadiens-français reproduit de larges extraits de l'encyclique Fidei Donum, reconnaissant la valeur de l'enseignement papal par rapport à l'avenir politique du continent ainsi que par rapport au rôle de leader que se doit de jouer l'Église catholique dans ce contexte de mutations majeures.

\section{MALGRÉ LA DIFFÉRENCE NATIONALE ET COLONIALE, DES PARCOURS PLUS SIMILAIRES QUE DISTINCTS...}

Cette troisième et dernière partie veut répondre à la question suivante : quelles similitudes et différences principales se dégagent de l'action et du discours des missionnaires belges et canadiens-français en Afrique au cours de la décennie 1950, tels qu'ils ont été exposés précédemment? Il me semble que cinq similitudes structurent les liens entre religion et politique, tels que nous les avons déclinés dans notre introduction (versant interne au catholicisme et versant externe des rapports entre Église et États) dans ce contexte africain partagé par les deux groupes missionnaires étudiés.

Premièrement, autant les Canadiens français que les Belges peuvent compter sur des médias solides (périodiques et films missionnaires), sur des réseaux d'intelligence missionnaire ainsi que des ressources financières en appui aux missions. D’ailleurs, ces réseaux d'intelligence des missions débouchent sur la fondation et le développement d'institutions diverses en mission (AUCAM, Université Lovanium, Aide intellectuelle des missions) qui favorisent la formation d'une élite africaine que l'on souhaite catholique. Rappelons que pour l'émergence de médias missionnaires et d'outils visant le développement de l'intelligence des missions, les Belges ont devancé les Canadiens français même si ces derniers, dans les années 1950, disposent d'infrastructures comparables à celles des Belges, dans le

70. Vincent de Decker, «La mission d'Afrique», Vivante Afrique, 196 (avril-mai 1958), 60. 
pays d'origine ainsi qu'en mission. Ces médias jouent une fonction idéologique majeure par la transmission d'un discours religieux faisant la promotion d'une action "civilisatrice», évangélisatrice et parfois colonisatrice (comme dans le cas des Belges) et favorisant le zèle missionnaire de leurs coreligionnaires, le recrutement de futur(e)s missionnaires occupant le haut du pavé des objectifs ainsi visés.

Deuxièmement, l'horizon de la mission est, tant dans le cas québécois que belge, celui de l'apport «civilisateur» à des peuples qui sont considérés comme inférieurs au monde euro-occidental sur les plans culturel, matériel, technique et moral, d'où l'intérêt de les convertir au catholicisme. D’une certaine manière, cette dimension de l'action missionnaire représente le bras culturel de la domination politique colonisatrice à laquelle sont soumises les nations africaines à l'époque. D'ailleurs, autant les Canadiens français que les Belges adhèrent à ce projet, selon des degrés divers suivant l'avancement de la décennie, les enjeux soulevés et la dynamique coloniale dans laquelle ils se situent. De plus, cette perspective doit permettre à l'Église catholique de se maintenir solidement implantée, peu importe les aléas propres aux changements politiques et sociaux qui pointent à l'horizon ainsi que les confrontations idéologiques avec l'Islam, le communisme, le paganisme et le protestantisme, à travers la formation d'une élite catholique qui devrait occuper les postes de responsabilité une fois les transformations opérées. Cependant, à la lumière de certains de ses effets débilitants sur les populations africaines, les Pères blancs canadiens-français s'interrogent sur la valeur positive de cette action "civilisatrice».

Troisièmement, les formes d'engagement missionnaire, que sont la santé, l'éducation, les presses missionnaires et les divers groupes inspirés de la doctrine sociale catholique et les structures d'appui aux missions étrangères belges et canadiennes-françaises, sont sensiblement les mêmes, sauf en cela que les premières sont financées par l'appareil colonial belge, ce dont ne bénéficient pas au même niveau les missions de Canadiens français $^{71}$. L'accent mis, dès le début de la décennie, sur la formation d'une élite à travers l'éducation témoigne de son importance dans l'implantation solide de l’Église catholique en sol africain. De plus, l'éducation primaire et secondaire en mission est le lieu, dans divers pays de l'Afrique centrale

71. Notons qu'il arrive que des missionnaires québécoises soient au service de sociétés coloniales, des entreprises exploitant les ressources naturelles, comme dans le cas du Congo belge. De plus certaines ressources financières peuvent être octroyées par d'autres puissances coloniales comme la GrandeBretagne, mais cela semble plus rare, selon ce que rapporte la revue canadienne-française des Pères blancs, de même que la littérature scientifique exposée sur le sujet. 
et orientale, de confrontations majeures avec le gouvernement colonial, ce que dénoncent autant les Belges que les Canadiens français.

Quatrièmement, autant les femmes missionnaires belges que canadiennes-françaises sont des subalternes par rapport à leurs confrères simples missionnaires, qu'ils soient frères ou pères. Elles font également les frais de la domination masculine dans les hautes sphères ecclésiastiques ainsi que sur le terrain, bien que leur situation s'améliore notamment du point de vue de leur accès à des études plus poussées sur les plans professionnel, pastoral et théologique même au cours de la décennie 1950. De plus, les femmes dans les missions catholiques sont reconnues comme une pièce centrale de la stratégie visant à s'intégrer aux communautés locales en entrant dans les familles. Toutefois, les femmes demeurent des exécutantes la plupart du temps sur le terrain missionnaire, leurs fonctions professionnelles étant reconnues comme valables dans la mesure où elles permettent de gagner la confiance des populations à évangéliser, mais l'Église n'accorde à leur fonction proprement religieuse aucune valeur monétaire ni symbolique, contrairement à celle des prêtres qui voient leur fonction sacerdotale être reconnue et rétribuée symboliquement (par exemple, par l'accession à l'épiscopat) et financièrement. Dans les événements publics où elles sont présentes, les paroles des religieuses ne sont pas rapportées, même si les enjeux soulevés concernent les femmes ou les filles comme dans le cas de la première rencontre des laïques africains de 1953.

Cinquièmement, le caractère supranational de la politique vaticane des missions a également contribué à faire admettre, parmi une majorité de missionnaires engagés en Afrique au cours des années 1950, et ce, peu importe leur appartenance nationale, la valeur positive du processus d'africanisation des Églises locales tout autant que celui, politique, de l'accession à l'indépendance des peuples africains. Cela est visible autant dans l'attitude de Rome que dans celle des épiscopats nationaux. De plus, à travers les encycliques relayées par les deux revues étudiées, le pape a contribué à la défense des intérêts de l'Église catholique sur le continent africain, notamment en rappelant l'importance de poursuivre l'œuvre "civilisatrice» dans le contexte des luttes idéologiques en cours et des bouleversements culturels et sociopolitiques annoncés. Autrement dit, Rome tentait de positionner l'Église catholique, par le biais de ses missionnaires et des œuvres poursuivies par ces derniers, comme une mère et une accompagnatrice, championne et défenderesse du droit des peuples africains à l'autodétermination politique. 
En contrepartie, une différence apparaît du point de vue de la distinction des rapports entre religion et politique en contexte missionnaire africain chez les Pères blancs belges et canadiens-français. Ce qui me semble avoir pu teinter les représentations de soi et de l'Autre des missionnaires belges et québécois ainsi que leur action en Afrique au cours de la décennie 1950 reste leur appartenance nationale et, par ricochet, l'adhésion ou la désaffiliation au fur et à mesure qu'avance la décennie, dans le cas des Belges, au projet politique proprement colonisateur de leur État national, spécialement dans le contexte du Congo belge. Le cas de l'éducation primaire et secondaire au Congo ainsi qu'au Ruanda-Urundi est en ce sens révélateur du passage qui s'opère alors dans la revue belge étudiée vers un détachement et une distinction plus grande des projets colonial et "civilisateur» missionnaire. Compte tenu de leur non-appartenance à une nation coloniale (sauf, de loin, à l'Empire britannique), les projets missionnaire et colonisateur pouvaient être plus aisément dissociés pour les Canadiens français. Les Pères blancs ne mentionnent qu'en de très rares occasions le système colonial dans lequel ils évoluent. De plus, dans leur manière d'appréhender le terrain missionnaire et d'en rendre compte dans leur publication, les Pères blancs canadiens-français tiennent davantage et plus tôt compte des divers défis qui se présentent sur le plan des confrontations idéologiques et politiques que leurs homologues belges. A contrario, ces derniers mettent davantage l'accent sur les liens entre le projet politique colonial et l'œuvre évangélisatrice et «civilisatrice » qu'ils réalisent comme missionnaires catholiques. C'est au moment où leurs œuvres d'éducation sont directement menacées par l'État colonial belge qu'ils commencent à prendre une distance plus grande par rapport au discours colonisateur.

\section{CONCLUSION}

Cette étude a permis de montrer que les cas missionnaires belges et canadiens-français sont plus proches que nous serions portés à le croire au premier abord, du fait de leur différence majeure sur le plan politique, soit celle d'appartenir ou non à une nation colonisatrice dans le contexte missionnaire africain des années 1950. Tant par leurs actions que par leurs discours, les missionnaires canadiens-français et belges s'inscrivent d'abord dans une vision "civilisatrice» du travail à réaliser auprès des peuples avec lesquels ils sont envoyés par l'Église pour vivre. Cette vision est alimentée, dans les deux cas, par l'enseignement de l'Église catholique (du pape principalement) et du côté belge, aussi par les liens qui unissent les mis- 
sionnaires à l'État colonial. L'appartenance nationale des Belges influence cette lecture "civilisatrice» qui se double d'un sens de la domination politique (colonialisme) s'articulant à travers l'allocation de ressources financières ainsi que d'une position hiérarchique forte au sein de l'organisation administrative coloniale. Cette influence tend cependant à s'amenuiser au fur et à mesure que se cristallise le conflit politique entre les missionnaires belges et l'État colonial au sujet de l'éducation et que gagne en importance la politique vaticane qui, elle, défend les intérêts supranationaux de l'Église catholique. Devant un terrain missionnaire chargé d'idéologies diverses qui confrontent directement le catholicisme, la formation des élites, qu'ont entreprise autant les missionnaires belges que canadiensfrançais, se révèle le remède tout indiqué, en phase avec les enseignements contenus dans les encycliques Evangelii Praecones (1951) et Fidei Donum (1957).

$\mathrm{Si}$, comme je l'ai montré, la politique vaticane supranationale des missions apparaît comme un facteur plus déterminant sur l'action et le discours missionnaires belges que la politique nationale coloniale, il serait pertinent de valider si la distinction nationale interne belge entre Flamands et Wallons a pu influencer le positionnement des uns et des autres par rapport à l'appareil colonial en contexte missionnaire. Il y aurait aussi lieu d'analyser de manière approfondie la façon dont les femmes et les hommes missionnaires, canadiens-français et belges, ont traité les enjeux politiques de cette décennie afin de vérifier si des différences existent entre les sexes quant à la lecture qu'ils font de ce contexte. De même, une étude élargie à d'autres instituts missionnaires belges et canadiens-français présents en Afrique au cours de cette décennie mériterait d'être entreprise. 